\title{
Psicoterapia en Atención Primaria: consejo interpersonal para la depresión
}

\author{
M. Diéguez Porres, B. Rodríguez VegA*, A. Fernández LiRiA** \\ Psiquiatra. SSM Leganés. Madrid. *Psiquiatra. HU La Paz. SSM Alcobendas. \\ Universidad Autónoma de Madrid. **Psiquiatra. Coordinador de los SSM del \\ Área 3 de Madrid. Universidad de Alcalá de Henares. Madrid
}

\section{RESUMEN}

En este trabajo se describen las líneas generales de actuación del médico de Atención Primaria ante uno de los trastornos de mayor prevalencia en su práctica diaria como es la depresión. Se expone el marco general de esta intervención, descrita minu ciosamente en el manual original de Klerman y co laboradores y que ha sido posteriormente adapta da al contexto de AP. Se describen los cuatro focos entorno a los que se articula la intervención: due lo, transiciones de rol, disputas interpersonales y déficits interpersonales, aportando ejemplos de cada uno de ellos procedentes de la práctica clínica.

Palabras clave: Depresión. Terapia interperso nal. Atención Primaria.

\section{Psychotherapy in Primary Health Care: interpersonal counselling for depression}

Key words: Depression. Interpersonal therapy. Primary Health Care.

In this work we review the general principles of Primary Health Care counselling for depression as it is described in the Primary Health Care version of the original Interpersonal Therapy for Depres sion Gerard Klerman's manual. We review the four intervention focus, that is: grief, role transitions, interpersonal disputes and interpersonal deficits and we bring clinic vignettes extracted from the clinical practice.

\section{INTRODUCCIÓN}

El desarrollo actual de la red de Atención Primaria como primer filtro asistencial, facilita el hecho de que los pacientes que sienten cualquier tipo de malestar psíquico consulten en primera instancia a un médico general. La demanda de atención por este tipo de problemas es muy amplia y así lo prueban numerosos estudios sobre morbilidad psiquiátrica en Atención Primaria que sugieren cifras en torno al $20 \%{ }^{1-3}$. Buena parte de estos pacientes que presentan ansiedad, depresión o molestias somáticas funcionales nunca van a ser derivados a un servicio especializado y esto es así posiblemente por varios motivos. Por una parte se deben considerar los riesgos de "psiquiatrizar" a una población que en su mayoría no presenta un trastorno mental grave, sino un malestar relacionado con dificultades para adaptarse a determinados acontecimientos vitales estresantes. Además, la saturación de las consultas de especialistas y el excesivo coste que supone hace que, cada vez más, el peso del tratamiento de estos pacientes con "trastornos menores" recaiga en la Atención Primaria de salud. Más allá de las cifras, la experiencia de quienes desde los servicios de salud mental realizamos coordinaciones periódicas con Atención Primaria (AP), es que cada vez es mayor el interés de los médicos que tienen a su cargo este tipo de pacientes en adquirir habilidades de diagnóstico y trata- 
miento de aquellas personas que, de una u otro forma presentan dificultades emocionales o trastornos mentales establecidos.

No estaríamos hablando de psicoterapia si simplemente se tratara de saber cuándo y cómo utilizar determinados psicofármacos. Muchos de estos pacientes no demandan ni precisan un tratamiento farmacológico para superar sus síntomas que, por otra parte, no siempre aportan beneficios a largo plazo, sino encontrar formas de afrontar sus dificultades y de mejorar su funcionamiento psicosocial.

La psicoterapia es una alternativa terapéutica, incluso el tratamiento de elección en numerosos trastornos mentales, pero su práctica requiere la adquisición de una formación específica más propia de profesionales especializados. Sin embargo, la preocupación por este tema ha llevado al desarrollo de formatos de tratamiento psicoterapéutico especialmente pensados para ser aplicados en AP, adaptados a las características de formación de los profesionales y a las particularidades del encuadre. Es el caso, por ejemplo, de la propuesta de Balint quien, desde un modelo psicoanalítico, describió la técnica del "Flash" como una sugerente forma de aprovechar los momentos de "conexión con lo psicológico", que suceden en el contacto con los pacientes, para producir cambios en su funcionamiento previo. Es un modelo de intervención que no requiere tener conocimientos previos y que ha demostrado su eficacia en seguimientos a largo plazo. Más estandarizadas, incluso manualizadas, son la que describe Castelnuovo-Tedesco en La hora de veinte minutos $^{5}$ y la Terapia Interpersonal de Klerman et al. objeto de este artículo.

En este trabajo se describen las generalidades de esta última intervención manualizada que han sido adaptadas a las características del contexto de trabajo del médico de AP y que consideramos de gran utilidad para el adecuado manejo de uno de los trastornos psiquiátricos que con más frecuencia encuentra el médico de AP en su práctica cotidiana.

\section{CONSEJO O ASESORAMIENTO INTERPERSONAL EN ATENCIÓN PRIMARIA}

La terapia interpersonal para Atención Primaria (TIP-AP) ${ }^{6}$ es una intervención psicoterapéutica especialmente diseñada para pacientes que presentan síntomas de ansiedad o depresión en relación con acontecimientos vitales estresantes y que no tienen un trastorno mental grave. Se trata de un tratamiento breve (en torno a seis sesiones de 30 minutos) focalizado en el funcionamiento psicosocial actual del paciente y que puede ser practicado por profesionales de Atención Primaria previamente entrenados (médicos, enfermeros, trabajadores sociales). En ella se presta la mayor atención a cambios re- cientes en la vida del paciente, a situaciones de estrés emocional en el entorno familiar, laboral, etc., $\mathrm{y}$ a dificultades en las relaciones interpersonales. Se parte de la base de que tales acontecimientos actúan como factores desencadenantes o mantenedores de los síntomas psíquicos.

La TIP-AP se desarrolla a partir de la Terapia Interpersonal (TIP) creada por Klerman et al. en $1984^{7}$ para el tratamiento de la depresión y su eficacia ha sido probada en numerosos estudios científicos ${ }^{8-11}$. La intervención se caracteriza por: a) las estrategias; b) las técnicas y c) la actitud terapéutica. Está descrita en un manual en el que se precisan minuciosamente las actuaciones sesión a sesión.

El objetivo de la terapia interpersonal es reducir el estrés y los síntomas, mejorar el funcionamiento social $y$, en última instancia, reducir la utilización inapropiada de los servicios sanitarios. Esto se lleva a cabo mediante la identificación de los factores que contribuyen al estrés actual, que generan dificultades de adaptación y ayudando al paciente a encontrar estrategias más eficaces para afrontarlos.

\section{CARACTERÍSTICAS DE LA TIP-AP}

\section{Estrategias TIP}

Las estrategias son, en realidad, lo específico de la TIP. Supone el trabajo estructurado en tres fases: inicial, intermedia y de terminación

\section{Fases iniciales. Valoración}

La evaluación del caso concreto requiere una o dos sesiones. Durante esta fase se acometen cuatro tareas. La primera se intitula ocuparse de la depre sión. Una vez discutido con el paciente el resultado negativo de las pruebas físicas realizadas previamente, se lleva a cabo conjuntamente una revisión minuciosa de los síntomas depresivos para lo cual se pueden revisar directamente los resultados de cuestionarios de depresión y ansiedad. Dar un nom bre al síndrome e instruir al paciente sobre su pro blema y el tratamiento, nos permite otorgar al paciente el rol de enfermo. Durante esta fase se ha de evaluar la necesidad de medicación (cuyo uso conjunto está recomendado).

Ejemplo 1: "Así que Vd. me ha consultado por que desde hace tres meses, se encuentra sin ga nas de hacer sus tareas habituales y de mal humor con sus hijos, ha perdido ilusión por el futuro, le cuesta conciliar el sueño y no tiene ganas de comer. La exploración física y los datos de los análisis que le hemos hecho han resulta do ser normales, afortunadamente, pero las que jas que usted me refiere se corresponden con un 
trastorno bien definido como es la depresión. La depresión tiene hoy tratamiento, tanto psicofar macológico, si vemos que en su caso lo precisa, como psicoterapéutico, que consiste, en términos generales, en discutir con un experto los posi bles factores que estén condicionando la depre sión”.

Es el momento de llevar a cabo la segunda tarea que consiste en señalar la posible relación de los síntomas con acontecimientos vitales y conflictos en las relaciones interpersonales y de ofrecer la oportunidad de recibir ayuda para superarlos. Para esto se procede a revisar exhaustivamente, con el paciente, las características de sus relaciones interpersonales significativas (presentes y pasadas por este orden), las circunstancias vitales actuales y los cambios sucedidos en ellas recientemente para relacionarlas con los síntomas depresivos según una sistemática minuciosamente descrita en el manual.

Algunos ejemplos (ejemplo 2) de preguntas que ayudan a llevar a cabo esta tarea son: " ¿ha ha bido en los últimos meses algún cambio signifi cativo en su vida?", "hábleme de su familia... ¿quiénes la componen?”, “¿cómo es la relación con su marido? hábleme de él”, "en toda rela ción hay aspectos positivos y otros negativos... ¿qué cosas le gustan más de la relación con su marido?, ¿qué aspectos le gustan menos?, ¿qué cosas cambiaría de esa relación?, ¿qué le gusta ría mantener o mejorar?". "Hemos hablado mu cho de su relación actual con su marido y las di ficultades por las que atraviesa, ahora me gustaría que me hablase usted de otras relacio nes significativas en su vida”, “ ¿cómo fue la re lación con su padre?, ¿qué le gustaba más de él?, ¿qué menos?, ¿en qué cosas cambió esa re lación?", "dice usted que la relación con su padre se enfrió después de que usted se casase con una persona que a él no le gustaba, también que pronto empezaron las discusiones con su marido $y$, parece que es desde entonces, cuando empezó usted a sentirse decaída y falta de ilusión...".

La información recabada nos permite acometer la tercera tarea que trata de reformular el malestar del paciente en una de las cuatro áreas problema que se describen más adelante y sobre la que se va a trabajar: duelo, disputas interpersonales, transiciones de rol y déficits interpersonales. De acuerdo con esto, se plantean los objetivos del tratamiento.

Ejemplo 3: "Por todo lo que hemos estado ha blando en estas dos ocasiones en que nos hemos visto, me parece que las dificultades en la rela ción con su marido y las discusiones continuas en los últimos meses tienen una gran relación con su estado de ánimo triste. Le propongo que nos centremos especialmente en este conflicto en la relación con su marido, ayudarla a entender lo que está sucediendo entre ustedes y a renego ciar con él una relación diferente más satisfacto ria para ambos".

La última de las tareas acometidas en esta fase supone explicar la forma de trabajo conjunto y hacer un contrato terapéutico con el paciente. Éste es un elemento esencial en la TIP. En el contrato se deben explicitar: el/las áreas problema, los objetivos del tratamiento, el lugar, frecuencia y duración de las visitas y la actitud a tomar ante las vicisitudes previsibles mientras dure el tratamiento.

Ejemplo 3: "Según lo que hemos estado hablan do en estas dos últimas consultas, mi impresión es que su depresión tiene que ver con la separa ción aún reciente de su marido. Yo le propongo explorar un poco más en profundidad sus senti mientos y emociones tras esta separación, así como las dificultades por las que está usted atra vesando ahora. Lo podemos hacer viéndonos en esta consulta durante cinco o seis visitas sema nales de media hora de duración y que tendrán como objetivo revisar conjuntamente todo lo ocurrido en relación con este acontecimiento tan importante en su vida y ayudarla a poner en marcha de nuevo sus propias fuerzas y recursos. Si por cualquier motivo no pudiera usted venir, le ruego que me llame para anular la cita. Yo ha ría lo mismo en caso de que la dificultad me sur giese a mí. ¿Qué le parece?, ¿está usted de acuerdo?, ¿necesita alguna aclaración?"

\section{Fases intermedias}

El supuesto básico de la TIP está en considerar que los cuadros depresivos que van a tratarse con ella tienen que ver con problemas que se manifiestan en una de las cuatro áreas problema siguientes: a) duelo; b) disputas interpersonales; c) transiciones de rol; o d) déficits interpersonales (soledad y aislamiento social). Estos problemas no son mutuamente excluyentes y es posible que aparezcan dificultades en varias de estas áreas, pero en una terapia se trabaja en una, o en todo caso en dos de ellas, que se seleccionan de acuerdo con el paciente en las fases iniciales.

Para facilitar el trabajo se puede pedir al paciente que realice determinadas tareas fuera de las sesiones como hacer escritos, revisar fotografías, poner en práctica con las personas significativas habilidades adquiridas durante el tratamiento, etc.

Duelo. Se selecciona esta área problema cuando existe evidencia de dificultades en la elaboración de 
un duelo. Los objetivos de este trabajo son: facilitar el proceso de duelo y capacitar al paciente para restablecer el interés y las relaciones para sustituir lo perdido. Esto se consigue a través de una serie de estrategias que incluyen: a) una revisión de los síntomas depresivos; b) relacionar los síntomas con la muerte de la persona significativa; c) una reconstrucción de la relación del paciente con el fallecido; d) una revisión de la secuencia y consecuencias de los acontecimientos inmediatamente anteriores, durante y después de la muerte; e) una exploración de los sentimientos (positivos y negativos) asociados; y f) una consideración de las posibles maneras de entablar relaciones con otros.

Ejemplo 4: Joaquín es un paciente de 23 años que en el transcurso de una mañana de trabajo normal como impresor, comienza a presentar fuertes mareos. Tras varios días con esta sinto matología fue dado de baja laboral y se inició un estudio neurológico que, una vez finalizado, no reveló alteraciones a ese nivel. Su ánimo no era triste sino aparentemente preocupado por la posibilidad de padecer un tumor cerebral. Fue derivado a Psiquiatría dos meses más tarde tras haber presentado una crisis de angustia, inter pretada por el médico de cabecera como conse cuencia de la tensión nerviosa que el paciente sufría ante el TAC craneal que unos dias más tarde estaba previsto.

Al inicio de la entrevista, el paciente estaba muy inquieto, ante la posibilidad de la enfermedad fí sica. Relata sus sintomas del día anterior como dificultad respiratoria y sensación de muerte in minente. Cree que está preocupado como estaría cualquier otro en su situación, pues no se consi dera una persona aprensiva. Al interrogar por cambios en las relaciones significativas en los últimos tiempos, Joaquín relata la muerte de su novia diez meses antes. Tres años antes había si do diagnosticada de un tumor de piel. Había abandonado las revisiones, considerándose, por su cuenta, curada. Un año antes de su muerte, comenzó a presentar mareos, a los que su médi co de cabecera no concedió especial importan cia. Ante el agravamiento de la sintomatología se le hizo un TAC craneal que demostró la exis tencia de múltiples metástasis. Hasta la muerte de su novia, Joaquín se hizo cargo totalmente de su tratamiento y de la relación con los médicos, la familia de su novia se apoyó también en él evitando una tarea que les resultaba muy peno sa. Los últimos meses habian sido de una gran sobrecarga para Joaquín, que simultaneaba el trabajo en su empresa con el cuidado de la no via cada día más invalidada y consumida. Des pués de la muerte de su novia, Joaquín confiesa un sentimiento de gran liberación. Tras cumplir con todos los ritos, contactó de nuevo con sus amigos y se propuso volver a la vida normal. Sa lía todos los días, buscaba compañia de mujeres tanto dentro de su entorno como de prostitutas y comenzó a beber abusivamente. El mismo se sorprendía de "lo bien que había reaccionado a la muerte de su novia”. Sus amigos y su familia también. Unos meses más tarde comienza con el cuadro de mareos. Durante las primeras entre vistas fue capaz de ir conectando sus sintomas con los sentimientos no elaborados tras la muer te de su novia. Sólo después de llevar a cabo es ta tarea de identificación y revisión de senti mientos, conectándolos con su estado de ánimo actual, puede, el paciente iniciar el trabajo de duelo que incluirá también la revisión de la rela ción con su novia, con sus aspectos positivos y negativos y, más adelante, la reorganización o adquisición de nuevas relaciones en su vida.

Disputas interpersonales. Se selecciona cuando pueden identificarse situaciones interpersonales en las que existen expectativas no recíprocas entre el paciente y otra persona significativa y pueden relacionarse con la sintomatología depresiva. Los obje tivos son: a) identificar la disputa; b) escoger un plan de acción y c) o modificar los patrones de comunicación o revaluar las expectativas o ambas cosas a la vez. El terapeuta no tiene por qué guiar al paciente hacia ninguna solución en particular y no intenta mantener relaciones imposibles.

En cuanto a las estrategias se revisan: a) los sín tomas depresivos; b) se ponen en relación con la disputa abierta o encubierta con otro significativo con el que el paciente está comprometido y c) se determina la fase en la que está actualmente la dis puta. Si la disputa está en fase de impasse se tratará de llevarla a una fase de renegociación; si está en fase de disolución ésta se trabajará como un duelo.

Se trata de ayudar al paciente a entender cómo las expectativas no recíprocas se relacionan con la disputa e iniciar los pasos que pueden conducir a resolución de la disputa y negociación de roles.

Se trabajan para ello tanto aspectos prácticos (motivos, expectativas, valores, posibilidades, recursos, perpetuantes,...), como aspectos paralelos con relaciones previas o consecuencias de un número de alternativas antes de pasar a la acción.

Ejemplo 5: Ana consulta por sentimientos de tristeza y desesperanza que le llevaron a hacer un intento autolítico unos días antes, tras una fuerte discusión con su marido. Ana describe la relación con su marido como muy buena, desde su punto de vista, hasta unos meses antes, en que el marido, Alfonso, empezó a mostrarse irri table y hostil hacia ella y su familia de origen, sin que ella consiguiese ninguna explicación por 
aquello. Hace unos días, Alfonso le confesó la ruina económica de su negocio que les afecta a ellos como pareja y se extiende a la familia de Ana. Ella se sintió profundamente traicionada y no entiende cómo Alfonso no la tenía al tanto de la difícil situación por la que atravesaba. Según refiere el marido le reprochó no darse cuenta de ello al verle tan irritable y encerrado en sí mis mo y la acusó de "echarle a él toda la responsa bilidad económica y no querer enterarse cuando las cosas van mal". El terapeuta le propone a la paciente centrarse en cómo este conflicto en la relación con su marido está afectando a su esta do de ánimo y a entender cómo las expectativas no recíprocas de cada uno de ellos les llevaron a su situación actual de falta de comunicación.

Transiciones de rol. Se asocian a la depresión cuando la persona tiene dificultades para afrontar los cambios requeridos por el nuevo rol (padre, trabajador, jubilado,...). Las transiciones suelen asociarse a progresiones en el ciclo vital. Las dificultades se suelen acompañarse de pérdida de apoyos y vínculos familiares y sociales, manejo de las emociones concomitantes como rabia o miedo, exigencia de nuevo repertorio de habilidades o baja autoestima.

Los objetivos del trabajo en este área son: a) facilitar el duelo y aceptación de la pérdida del antiguo rol; b) propiciar una visión más positiva del nuevo y c) restaurar la autoestima desarrollando un sentimiento de dominio de los nuevos roles.

Las estrategias para el trabajo en este área comprenden: a) una revisión de los sintomas depresi vos; b) relacionar los síntomas depresivos con las dificultades para desenvolverse frente a algún cam bio vital reciente; c) una revisión de los aspectos positivos y negativos de los roles antiguos y nue vos; d) una exploración de los sentimientos ante lo que se ha perdido; e) ante el cambio mismo, y f) una exploración de las oportunidades que brinda el rol nuevo; g) se trata de evaluar realistamente lo perdido; h) animar una adecuada liberación de afectos e i) propiciar el desarrollo del sistema de apoyo y nuevas habilidades requeridas por el nue vo rol.

Ejemplo 6: Ramón es un hombre de 67 años que ha dedicado una gran parte de tiempo y esfuerzo en su vida a sacar adelante su pequeño negocio. Hace año y medio, desde que se jubiló, se siente triste y sin ilusión por la vida. El terapeuta le propone una serie de sesiones en las que se cen tren en hablar lo que significó para él la jubila ción. Ramón expresó los sentimientos asociados de inutilidad e infravaloración tras dejar el tra bajo, junto con todo lo que éste le aportaba de positivo. El terapeuta le ayudó en las siguientes sesiones a ver también algunos aspectos negati vos de su trabajo, de los que ahora se había li berado y a empezar a pensar en sí mismo como la persona valiosa que era, enfrentando una nueva etapa que requería de actividades diferen tes. Ramón se informó de algunas asociaciones de empresarios jubilados que ayudaban a otros más jóvenes asesorándolos para iniciar su acti vidad laboral. Encontró ahí una forma de orga nizar su tiempo libre a través de una actividad que le reforzaba su sentimiento de autoestima maltrecho tras la jubilación. Este paciente había sufrido un episodio depresivo al atravesar la transición a una fase distinta del ciclo vital. Por su historia biográfica y sus peculiaridades, esta transición suponía para él un fuerte ataque a su autoestima, construida a partir de ser la perso na activa y proveedora de cuidados materiales a su familia. A través de la intervención terapéuti ca, el paciente pudo empezar a disfrutar de otras áreas de la vida que tenía abandonadas por su dedicación a su negocio y abrir actividades nue vas que le ayudaban a reconstruirse como la persona valiosa que era.

Soledad y/o aislamiento social. Se eligen como foco de la TIP cuando un paciente presenta una historia de empobrecimiento social que incluye relaciones interpersonales inadecuadas o insuficientes. Son personas que nunca han establecido una relación duradera o continua como adultos. Suelen ser los pacientes más graves y, desde luego, los más difíciles de trabajar con esta técnica.

Al no existir relaciones actuales a las que referirse el foco se centra en relaciones pasadas o el comienzo de nuevas relaciones.

El trabajo en este área, particularmente ardua, atiende a los objetivos de: reducir el aislamiento social del paciente y animar la formación de nue vas relaciones. Para ello utiliza las siguientes estra tegias: a) revisar los síntomas depresivos; b) rela cionar los síntomas depresivos con los problemas de aislamiento o desengaño social; c) revisar las relaciones significativas del pasado, incluyendo los aspectos positivos y negativos; d) explorar pautas repetitivas en las relaciones pasadas o fracasadas; e) analizar los sentimientos positivos y negativos del paciente hacia el terapeuta (que es frecuentemente en estos casos la única relación vigente) y buscar paralelos en otras relaciones anteriores o en curso.

Ejemplo 7: Sara es una mujer de 37 años que nunca ha tenido una actividad laboral y que tras la muerte de su madre hace dos años, único familiar con el que convivía, se encuentra profun damente triste y abatida. Sara no tiene amigos ni otros familiares. Se describe como una persona 
muy tímida, con escaso aprecio por sí misma, a quien siempre le ha costado hacer amigos. La terapeuta le propone centrarse en ayudarla a elaborar el duelo tras la muerte de su madre, pero también en sus dificultades para establecer relaciones y en sus sentimientos de soledad y aislamiento asociados. La relación terapéutica se convierte en muchas ocasiones en el foco de discusión, al carecer la paciente de otras rela ciones significativas, pero la terapeuta no pierde de vista uno de los objetivos acordados y anima a Sara a matricularse en un curso que le puede ayudar a iniciar una actividad laboral. Al prin cipio, la paciente toma esta decisión, para ella muy dificultosa, en el contexto de la relación con la terapeuta, que se ha convertido en su persona de referencia, pero el propio curso se convierte en una fuente proveedora de red social y de al gunos sentimientos positivos de autoestima que son discutidos y aprovechados en la terapia.

\section{Fase de terminación}

Muchos pacientes pueden haber mejorado considerablemente antes de terminar las sesiones programadas y no se debe forzar su consecución si esto sucede con el fin de prevenir la dependencia y la sobreutilización de los servicios. La terminación debe ser abordada explícitamente en la sesión anterior de producirse el cese de la terapia. Para facilitar la tarea de terminación, al menos en la última entrevista se debe revisar lo que ha sucedido durante la terapia y el estado actual del paciente. Se debe reconocer esta terminación como un momento de posible duelo, llamar la atención del paciente sobre sus logros y reforzar su autonomía. En este momento conviene favorecer reacciones emocionales $\mathrm{y}$, a la vez, prevenir su interpretación como recaídas.

Si el paciente no se siente capaz de terminar se le puede ofrecer una sesión más pero dejando transcurrir un periodo de dos semanas para comprobar que verdaderamente lo necesita. En esta sesión se refuerza la idea de que será la última y se acometen las mismas tareas que en la anterior.

Ejemplo 8: "Sara, esta es la penúltima de las entrevistas que habiamos acordado, ¿lo recuer da?. Durante estas sesiones hemos hablado mu cho de lo que significó para usted la muerte de su madre y de cómo las dificultades para esta blecer relaciones nuevas la habian sumido en una situación de soledad y aislamiento, así que al empezar este tratamiento se encontraba usted profundamente triste. A través de todo este tiem po y de todo lo que hemos hablado y de todas las decisiones que usted ha ido tomando, se ha ido encontrando progresivamente mejor, hasta el punto de que creo que está usted en condiciones de continuar usted sola adelante en su vida man teniendo y acrecentando lo que ha iniciado en la terapia. Es posible que al principio de abando nar el tratamiento y dejar de vernos se pueda sentir usted de nuevo insegura de sus fuerzas o algo triste. Es normal que eso ocurra y no tiene por qué significar una recaída depresiva, sino una reacción normal al dejar una relación que a usted le ha servido de ayuda. Le animo a que si gamos hablando de ello en la próxima sesión, que será la última, y a que recuerde que si en el futuro lo volviese a necesitar podrá usted pedir consulta de nuevo".

\section{Técnicas}

El desempeño de este tipo de tratamientos requiere el dominio de algunas técnicas generales de entrevista y manejo clínico y el de algunas técnicas específicas. Sin embargo es importante puntualizar que a la TIP la caracterizan sus estrategias, no sus técnicas.

El manual describe las técnicas específicas utilizadas en la TIP, agrupadas en 7 grandes grupos: a) técnicas exploratorias; b) propiciación de los afectos; c) clarificación; d) análisis comunicacional; e) uso de la relación terapéutica; f) modificación de conducta y g) técnicas auxiliares (contrato, encuadre,...).

\section{Actitud terapéutica}

El manual define aquellos aspectos de la actitud terapéutica que diferencian la TIP de otras intervenciones psicoterapéuticas. Son los siguientes: a) el terapeuta actúa como abogado del paciente, no es neutral; b) la relación terapéutica no es una relación de amistad, se puede ser próximo pero no un amigo fuera de las sesiones y c) el terapeuta es activo a la hora de ayudar al paciente a hablar de sus dificultades y en la realización de tareas y puesta en práctica de logros.

\section{EL TERAPEUTA TIP: FORMACIÓN}

Como ya señalamos anteriormente, la TIP-AP puede ser practicada por cualquier profesional de Atención Primaria entrenado (médicos, enfermeros, trabajadores sociales).

A la formación se accede a través del estudio de material escrito (el manual) que se completa con la asistencia a un seminario didáctico en el que se trabaja sobre material audiovisual y se hace espe- 
cial énfasis en los cuatro aspectos en los que se han detectado dificultades con más frecuencia: a) reconocimiento de la depresión como un trastorno médico; b) énfasis en los problemas actuales de funcionamiento interpersonal; c) carácter exploratorio del proceso de tratamiento (el manual no es un libro de cocina) y d) carácter breve de la intervención. Se requiere, además, la supervisión de varios casos. Se trata de una supervisión semanal, sesión por sesión que puede hacerse individualmente o en grupos.

La TIP-AP se presenta como un acercamiento psicoterapéutico útil para ser puesto en marcha en Atención Primaria, del que pueden beneficiarse los pacientes y los médicos al conseguir sus objetivos terapéuticos y, en general, la Medicina al incluir en su práctica intervenciones psicosociales que ayudan a poner en marcha, y no sólo a pensar, una visión integral del ser humano.

\section{CORRESPONDENCIA:}

M. Diéguez Porres

Centro de Salud Mental de Leganés

$\mathrm{P}^{\mathrm{o}}$ de Colón, $\mathrm{s} / \mathrm{n}$

28911 Leganés

Madrid

\section{Bibliografía}

1. Üstün TB, Sartorius N. (Ed) Mental Illness in General Health Care. Chichester: John Wiley \& sons. (WHO), 1995.

2. Goldberg D, Huxley P. Common Mental Disorders: a biosocial model. London: Routledge, 1992.

3. Vázquez-Barquero J, Diéz-Manrique JF, Peña C, Aldama J, Samaniego Rodríguez C, Menéndez Arango J, et al. A community mental health survey in Cantabria: a general descrption of morbidity. Psychological Medicine 1987; 17: 227-41.

4. Balint E, Norell JS. Seis minutos para el paciente. Buenos Aires: Paidos, 1979.

5. Castelnuovo-Tedesco P. The twenty-minute hour: A guide to brief psychotherapy for the physician. Washington DC: American Psychiatric Press, 1986.

6. Klerman GL, Weissman MM. Interpersonal counseling for Stress and Distress in Primary Care Settings. En: Klerman GL, Weissman MM, editores. New aplications of interpersonal psychotherapy. Washington: American Psychiatric Press 1993; 295-319.
7. Klerman GL, Rousanville B, Chevron E, Neu C, Weissman MM. Interpersonal psychotherapy of depression (IPT). New York: Basic Books, 1984.

8. Weisman MM, Prusoff BA, Dimascio A, Neu C, Gohlaney M, Klerman GL. The efficacy of drugs and psychotherapy in the treatment of acute depresive episodes. Am J Psychiatry 1979; 136: 555-8.

9. Elkin I, Shea T, Watkins JT, Imber SS, Sotsky SM, Collins JF, et al. National Institute of Mental Health Treatment of Depresion Collaborative Research Program: general effectiveness of treatment. Archives of General Psychiatry 1989; 46: 971-82.

10. Klerman GL, Dimascio A, Weissman MM, Prusoff BA, Paykel ES. Treatment of depresion by drugs and psychotherapy. Am J Psychiatry 1974; 131: 186-91.

11. Klerman GL, Budman S, Berwick D, Weissman MM, Damico-White J, Demby A, et al. Efficacy of a brief psychosocial for symptoms of stress and distress among patients in primary care. Medical Care 1987; 8: 6-15. 